\title{
Primary CNS Marginal Zone Lymphoma: A Case Report and Review of the Literature
}

\author{
Barina Aqil $^{1}$, Emilie Rouah ${ }^{1,2}$, Gordana Verstovsek ${ }^{1,2}$ \\ ${ }^{1}$ Department of Pathology \& Immunology, Baylor College of Medicine, Houston, USA; ${ }^{2}$ Department of Pathology, Michael E. De- \\ Bakey VA Medical Center, Houston, USA. \\ Email: aqil@bcm.edu
}

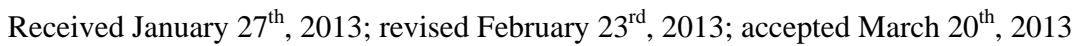

Copyright (C) 2013 Barina Aqil et al. This is an open access article distributed under the Creative Commons Attribution License, which permits unrestricted use, distribution, and reproduction in any medium, provided the original work is properly cited.

\begin{abstract}
Primary central nervous system lymphoma (PCNSL) is a rare subtype of extranodal non-Hodgkin lymphoma (NHL) located in the craniospinal axis (affecting CNS, meninges and eye) without evidence of a systemic primary tumor. CNS lymphomas are typically high grade, large cell lymphomas; low grade CNS lymphomas are rare. We encountered a case of 48-year old patient with recurrent episodes of seizures, weakness of bilateral extremities and memory loss. On MRI and CT scan, a $2.0 \mathrm{~cm}$ area of ill-defined enhancement with associated edema was present in the left frontal subcortex. The biopsy revealed an infiltrate of small lymphocytes expressing pan B-cell markers and an immunoprofile consistent with an extranodal marginal zone B cell lymphomas of mucosa associated lymphoid tissue (MALT). Clonal rearrangement was demonstrated by PCR analysis. Subsequently, the patient was treated with radiation therapy and responded well, with decrease in the size of the lesion. He is alive and well for 1 year and 3 months up to the date of this report.
\end{abstract}

Keywords: Primary CNS Lymphoma; MZL

\section{Introduction}

PCNSL are a rare type of NHL constituting $2.7 \%$ of primary brain tumors [1,2], and $0.7 \%-1.7 \%$ of NHL [3]. They mainly involve the leptomeninges, brain parenchyma, eyes and spinal cord, and are mostly found as duralbased masses mimicking meningioma [1]. Almost all PCNSLs are aggressive neoplasms, with diffuse large B cell lymphoma type comprising 95\% [1,4]. Primary low grade CNS lymphomas have much lower incidence, most of them described in the literature as small series or single case reports, and have an indolent course and a much better prognosis than large cell lymphomas.

Isaacson and Wright [5] first described marginal zone lymphoma (MZL) of the gastrointestinal tract as a low grade lymphoma. Since then it has been described in many other organs, including lung, bladder, salivary gland, lacrimal gland, thyroid, breast, skin and CNS. In the current World Health Organisation (WHO) classification, MZL is divided into extranodal mucosaassociated lymphoid tissue (MALT) lymphoma, nodal MZBL and splenic MZBL [6]. Prognosis of MZL is usually favorable, with MALT lymphoma usually presenting as stage I or II disease and running an indolent course [6].
MALT type of MZL in the CNS is a rare entity. CNS MALT lymphomas are low grade and localized lesions in contrast to CNS metastasis of the systemic lymphoma. Initial case reports and case series described MZL arising most frequently from the meninges. $93 \%$ cases in the series described by $\mathrm{Tu} \mathrm{Ph}$ et al. [7], leading to their inclusion in the differential diagnosis with meningioma. Other case reports describe less common parenchymal MZL which can be confused with gliomas. Herein, we present a case of CNS MALT lymphoma arising in the brain parenchyma of frontal subcortex, which was thought initially to be a high grade glioma.

Based on the immunohistochemistry and molecular studies the tumor was classified as a low grade lymphoma, most consistent with MZL lymphoma. Interpretation of an intraoperative brain biopsy can be challenging due to scant lesional cells and/or artifactual distortion of lesional tissue. Awareness of this rare entity is important so that appropriate handling of the specimen can be done. We present a case of a low grade PCNS lymphoma, with immunohistochemical and molecular profile, and review the literature on low grade CNS lymphomas. 


\section{Case Report}

The patient was a 48-year-old HIV-negative male who presented with five months history of recurrent generalized tonic-clonic seizures, weakness and numbness of bilateral upper extremities, more prominent on the right side. He had been on dilantin without much improvement. His only other significant complaint was long standing polyarticular arthritis.

CT scan, as well as MRI revealed a $2.0 \times 1.4 \times 1.6 \mathrm{~cm}$ area of ill-defined enhancement in the left frontal subcortex with extensive edema predominantly involving the left frontal subcortex and to a lesser degree left temporal and parietal subcortices [Figure 1(a)]. No midline shift was noted. The clinical impression was that the lesion most likely represented a glioma, and less likely cerebritis or a lymphoma. Pertinent laboratory values included: WBC $6.4 \mathrm{~K} / \mathrm{cmm}$ (normal: 3.5 - 10.0); RBC 4.1 $\mathrm{M} / \mathrm{cmm}$ (4.0 - 5.9); Platelet count $135 \mathrm{~K} / \mathrm{cmm}$ (150 450); Neutrophils 75.7\% (41.0 - 78.0); Lymphocytes 14.3\% (15.0 - 47.0); Monocytes 7.1\% (2.0 - 10.0); Eosinophils $2.1 \%$ (0.0 - 8.5); Basophils $0.8 \%$ (0.0 - 2.0); Rheumatoid factor $22.9 \mathrm{IU} / \mathrm{mL}$ ( 0 - 20); Sedimentation rate 30 $\mathrm{mm} / \mathrm{hr}(0-10)$.

Histologic evaluation of the biopsied subcortical lesion showed prominent perivascular lymphocytic infiltrates composed of small to intermediate sized lymphocytes, most of which had irregular nuclear borders and scant to moderate amount of cytoplasm.

Immunohistochemistry showed the lymphoid cells to be positive for CD20 and BCL2, but negative for CD5, CD10, CD23, CD43 and Cyclin D1. Ki-67 proliferation index was also very low (2\% - 5\%) [Figures 2(A) and (B)]. Due to the small sample size, flow cytometry could not be performed. Clonal rearrangement was demonstrated by PCR analysis using primers to framework 2 and 3 region of the immunoglobin heavy chaingene. Fluorescence in situ hybridization for IGH/BCL2 and IGH/ CCND1 were negative. Based on morphology, immunophenotype and molecular studies, a diagnosis of low grade lymphoma, most consistent with a MZL was rendered. A dural biopsy performed at the time of surgery showed no abnormality. Cerebrospinal fluid cytology was negative for malignant cells. Bone marrow biopsy with flow cytometry and cytogenetics showed no evidence of lymphoma. CT scan of the thorax, abdomen and pelvis had no evidence of systemic disease (specifically no lymphadenopathy). PET scan was remarkable for a small focus of increased signal, which corresponded to the lesion in left frontal lobe. Scrotal ultrasound was also negative.

The patient received whole brain external beam radiotherapy, with a total dose of 24 Gy after which the

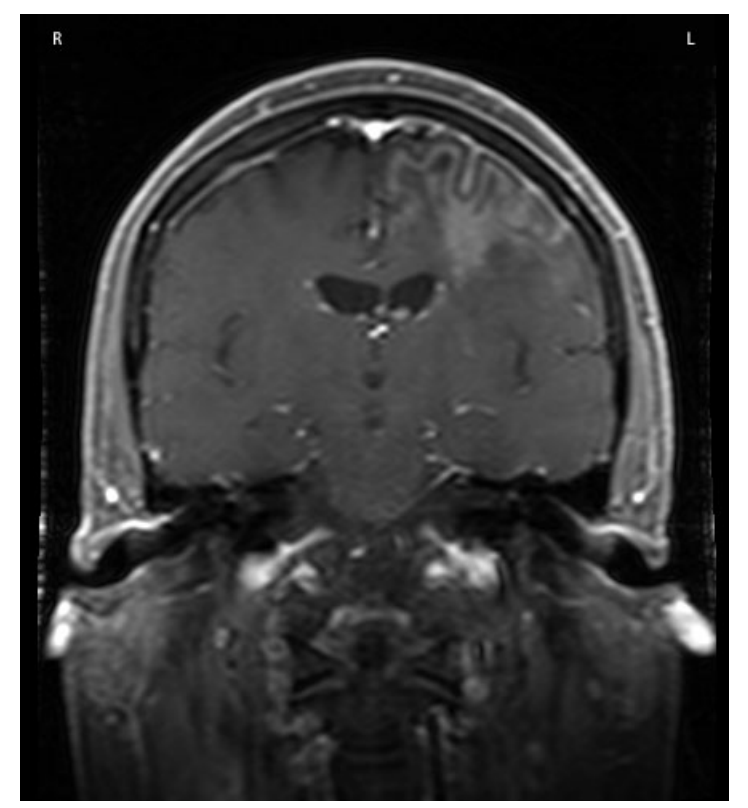

(a)

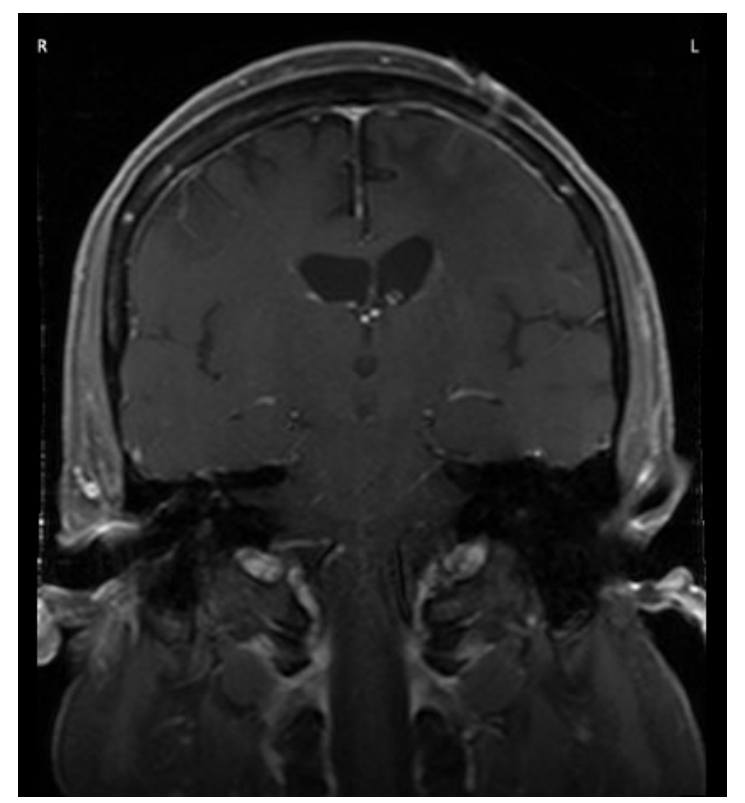

(b)

Figure 1. (a) MRI showed subcortical and deep white matter T2 hyperintensity present predominantly involving the left frontal subcortex and to lesser degree left temporal and parietal subcortices. The area of ill-defined enhancement measured $2.0 \times 1.4 \times 1.6 \mathrm{~cm}$; (b) Following radiation treatment imaging showed no mass effect or abnormal enhancement.

gross disease in the left frontal lobe was boosted by additional 6 Gy with a 3-field technique. Follow up MRI showed no mass effect or abnormal enhancement Figure 1(b); it did show persistent white matter changes which were thought to be radiation induced. Currently, he is 15 months from diagnosis and without seizures. 

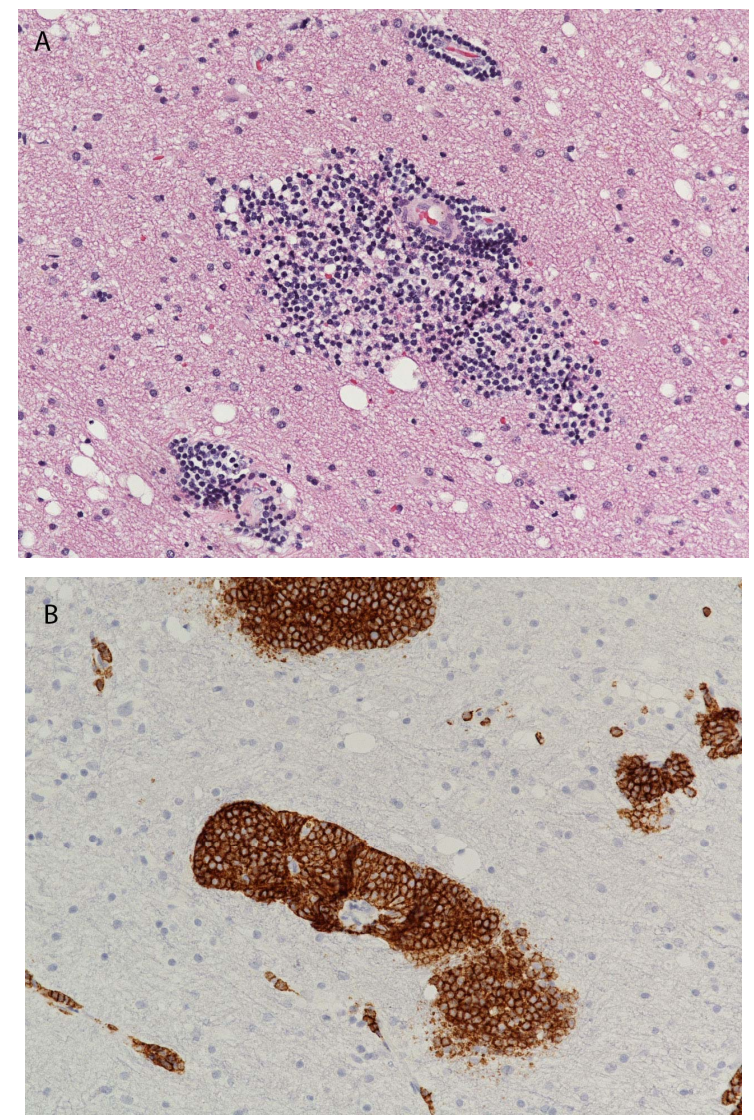

Figure 2. The brain biopsy showed perivascular infiltrates of small lymphocytes (A), which by immunohistochemistry proved to be CD20 positive B-cells (B) ((A) Hematoxilin \& Eosin, 200 $\times$; (B) Immunoperoxidase, 200 $\times$ ).

\section{Discussion}

Low grade CNS lymphoma is a rare entity. Among cases described in the literature, primary MZL has mostly been described in the extra-axial dura, with only 5 patients presenting with primary CNS MZL arising in the brain parenchyma, including our patient [7-10] [Table 1].

Our case is that of a 48 year old man presenting with seizures, and presumed clinical diagnosis of glioma. Biopsy demonstrated perivascular aggregates of small CD20 positive B-lymphocytes. Differential diagnosis included a small lymphocytic lymphoma/chronic lymphocytic leukemia (CLL/SLL), follicular lymphoma, mantle cell lymphoma and marginal zone lymphoma.

CLL/SLL was unlikely due to absence of peripheral blood or bone marrow involvement, and immunophenotype (CD5-, CD43-). The lymphocytes were negative for CD10 and Bcl-2 by immunohistochemistry as well as $\mathrm{Bcl}-2$ gene rearrangement by FISH, making follicular lymphoma unlikely. Similarly, negativity for Cyclin D1 and CD5 by immunohistochemistry, and for IGH/CCND1 by FISH ruled out mantle cell lymphoma. Therefore, based on exclusion of other types of low grade lymphomas,
Table 1. Case reports of patients reported with primary CNS MZL without dural involvement.

\begin{tabular}{|c|c|c|c|c|}
\hline Reference & Age/Sex & Location & Diagnosis & HIV \\
\hline Itoh, 2001 & $28 / \mathrm{F}$ & CP, Angle & MZCL & Unknown \\
\hline Tu et al. 2005 & $66 / M$ & $\begin{array}{l}\text { R, Frontal } \\
\text { cortex }\end{array}$ & MZCL & Unknown \\
\hline Park et al. 2008 & $18 / \mathrm{M}$ & $\begin{array}{l}\text { L, Basal } \\
\text { Ganglia }\end{array}$ & MZCL & Negative \\
\hline $\begin{array}{l}\text { Papanicolau } \\
\text { et al. } 2011\end{array}$ & 70/M & $\begin{array}{l}\text { L, Posterior } \\
\text { putamen }\end{array}$ & MZCL & Negative \\
\hline Aqil, 2012 & $48 / \mathrm{M}$ & $\begin{array}{l}\text { L, Frontal } \\
\text { cortex }\end{array}$ & MZCL & Negative \\
\hline
\end{tabular}

and confirmation of clonal rearrangement by PCR analysis using primers to framework 2 and 3 region of the immunoglobin heavy chain gene, a diagnosis of a PCNS lymphoma, consistent with extranodal marginal zone lymphoma (MALT lymphoma) was rendered.

The CNS contains neither mucosa nor MALT tissue. It has been hypothesized that meningothelial cells of the arachnoid membrane are analogous to epithelial cells in tissues from which MALT lymphomas arise. These cells are present throughout the arachnoid membrane and concentrated in the arachnoid granulations, but also in the choroid plexus and subarachnoid cisterns [11]. Therefore this theory correlated well with low grade CNS lymphomas arising extra-axially [12]. However, in our case the MALT tumor arose from the subcortex away from any meningothelial lining, the dural biopsy was normal, and the MRI did not show any tumor connection with the meninges, making meningothelial role in the pathogensis of this lymphoma questionable.

Outside the CNS, infectious and/or autoimmune processes are considered to be the etiologic agents for development of some MALT lymphomas. Such are chronic infection with Helicobacter Pylori (stomach) [13], Campylobacter jejuni (small bowel) [14], Chlamydia psittaci (ocular adnexal) [15], Borrelia burgdorferi (skin) [16]. Some autoimmune diseases are also reported including Sjogren's syndrome associated with salivary gland MZL [17] and Hashimoto thyroiditis with thyroid MZL [18]. Similarly, several reports have described the association of primary CNS lymphoma with Sjogren's syndrome [8], multiple sclerosis [7] and Graves disease [19]. Interestingly, our patient had a long standing polyarthritis, and based on laboratory values (mildly elevated rheumatoid factor and sedimentation rate) an underlying inflammatory process can be postulated, however to date it has not been defined, as multiple serologic tests in the patient failed to pinpoint the specific inflammatory disease (data not shown).

Genetic abnormalities have been identified in MZL, the most specific $t(11 ; 18)$ (q21;q21) or API2-MALT1 for the MALT type of MZBL translocation has been described [20]. Yet the most common abnormality is the 
Trisomy 3 in all type of MZL [21]. However API2MALT translocation has not been described yet for primary CNS MZBL. Tu et al. [7] analyzed the genetic abnormalities in 15 cases of primary CNS MZL and demonstrated Trisomy 3 by FISH in 50\% of the cases; similar to what had been demonstrated for MZL of other sites. BCL-6 proto-oncogene is located at $3 \mathrm{q}$ and has been linked with diffuse large B cell lymphoma [22]. BCL6 is involved in proliferation of germinal centers B cells. It is also suspected to be responsible for the pathogenesis of subset of MZBL with $t(3 ; 14)$ (q27;q32) [23]. Therefore Trisomy 3 was considered responsible for overexpression of BCL6 and development of CNS MZCL. However Tu et al. study could not demonstrate the over expression of BCL6 with presence of Trisomy 3.

Due to limited number of cases, treatment options for low grade CNS lymphomas have not been fully defined. Variable treatment options have been described ranging from surgery alone $[7,24]$ to radiation alone [12,24], or combined, with or without systemic chemotherapy [7, 12]. Radiation is considered superior to chemotherapy, as MZL is radiosensitive and remission can be achieved with a lower (20 - $45 \mathrm{~Gy}$ ) dose of radiation, therefore avoiding chemotherapy-induced neurotoxicity [19,2527]. High dose methotrexate is beneficial in high grade CNS lymphomas but not useful in low grade primary CNS lymphomas, unless there is leptomeningeal involvement by lymphoma [28]. Our patient received whole brain external beam radiotherapy of approximately $24 \mathrm{~Gy}$. He responded well to the treatment with reduction in the size of the lesion as well as improvement of his symptoms.

\section{Conclusion}

Low grade CNS lymphomas are rare and on biopsy demonstrate as small lymphocytic infiltrates, often in a perivascular fashion. Perivascular small lymphocytic infilltrates similar to those seen in this case can be seen in inflammatory and/or infectious process, as well as at the periphery of a large B-cell lymphoma. Therefore, awareness of this entity is important particularly when dealing with a small biopsy. Treatment and prognosis of CNS low grade lymphomas are significantly different from the more common high grade CNS lymphomas.

\section{REFERENCES}

[1] D. Raoux, S. Duband, F. Forest, B. Trombert, M. L. Chambonnière, J. Dumollard, A. Khaddage, A. GentilPerret and M. Péoch, "Primary Central Nervous System Lymphoma: Immunohistochemical Profile and Prognostic Significance,” Neuropathology, Vol. 30, No. 3, 2010, pp. 232-240.

[2] D. L. Commins, "Pathology of Primary Central Nervous
System Lymphoma,” Neurosurgical Focus, Vol. 21, No. 5, 2006, pp. E2.

[3] N. P. Mendenhall, T. L. Thar, O. F. Agee, et al., "Primary Lymphoma of the Central Nervous System. Computerized Tomography Scan Characteristics and Treatment Results for 12 Cases," Cancer, Vol. 52, No. 1983, pp. 19932000.

doi:10.1002/1097-0142(19831201)52:11<1993::AID-CN CR2820521104>3.0.CO;2-C

[4] N. L. Harris, E. S. Jaffe, H. Stein, P. M. Banks, J. K. Chan, M. L. Cleary, G. Delsol, C. De Wolf-Peeters, B. Falini and K. C. Gatter, "A Revised Europe and American Classification of Lymphoid Neoplasms: A Proposal from the International Lymphoma Study Group,” Blood, Vol. 84, No. 5, 1994, pp. 1361-1392.

[5] P. Isaacson and D. H. Wright, "Malignant Lymphoma of Mucosa-Associated Lymphoid Tissue. A Distinctive Type of B-Cell Lymphoma,” Cancer, Vol. 52, 1983, pp. 14101416.

doi:10.1002/1097-0142(19831015)52:8<1410::AID-CNC R2820520813>3.0.CO;2-3

[6] S. H. Swerdlow, E. Campo, N. L. Harris, E. S. Jaffe, S. A. Pileri, H. Stein, J. Thiele and J. W. Vardiman, "WHO Classification of Tumours of Haematopoietic and Lymphoid Tissues," 4th Edition, IARC Press, Lyon, 2008.

[7] P. H. Tu, C. Giannini, A. R. Judkins, J. M. Schwalb, R. Burack, B. P. O’Neill, A. T. Yachnis, P. C. Burger, B. W. Scheithauer and A. Perry, "Clinicopathologic and Genetic Profile of Intracranial Marginal Zone Lymphoma: A Primary Low-Grade CNS Lymphoma That Mimics Meningioma,” Journal of Clinical Oncology, Vol. 23, No. 24, 2005, pp. 5718-5727. doi:10.1200/JCO.2005.17.624

[8] T. Itoh, M. Shimizu, K. Kitami, K. Kamata, K. Mitsumori, M. Fujita, A. Ohnishi and K. Nagashima, "Primary Extranodal Marginal Zone B-Cell Lymphoma of the MucosaAssociated Lymphoid Tissue Type in the CNS," Neuropathology, Vol. 21, No. 3, 2001, pp. 174-180. doi:10.1046/j.1440-1789.2001.00392.x

[9] I. Park, J. Huh, J. H. Kim, S. W. Lee, M. H. Ryu and Y. K. Kang, "Primary Central Nervous System Marginal Zone B-Cell Lymphoma of the Basal Ganglia Mimicking Low-Grade Glioma: A Case Report and Review of the Literature,” Clinical Lymphoma Myeloma, Vol. 8, No. 5, 2008, pp. 305-308. doi:10.3816/CLM.2008.n.043

[10] A. Papanicolau-Sengo, J. Wang-Rodriguez, W. HuanYou, R. Lee, A. Wong, L. A. Hansen, S. Sepi Mahooti and H. H. Rashidi, "Rare Case of a Primary Non-Dural Central Nervous System Low Grade B-Cell Lymphoma and Literature Review," International Journal of Clinical and Experimental Pathology, Vol. 5, No. 1, 2012, pp. 8995.

[11] G. N. Fuller and J. C. Goodman, "Practical Review of Neuropathology,” Lippincott, Williams \& Wilkins, Philadelphia, 2001, pp. 57-58.

[12] S. Kumar, D. Kumar, E. P. Kaldjian, et al., "Primary Low-Grade B-Cell Lymphoma of the Dura: A Mucosa Associated Lymphoid Tissue-Type Lymphoma," The American Journal of Surgical Pathology, Vol. 21, No. 1, 1997, pp. 81-87. 
doi:10.1097/00000478-199701000-00009

[13] A. C. Wotherspoon, C. Doglioni, T. C. Diss, et al., "Regression of Primary Low Grade B-Cell Gastric Lymphoma of Mucosa-Associated Lymphoid Tissue Type after Eradication of Helicobacter Pylori," Lancet, Vol. 342, 1993, pp. 575-577. doi:10.1016/0140-6736(93)91409-F

[14] M. Lecuit, E. Abachin, A. Martin, et al., "Immunoproliferative Small Intestinal Disease Associated with Campylobacter Jejuni," The New England Journal of Medicine, Vol. 350, No. 8871, 2004, pp. 239-248. doi:10.1056/NEJMoa031887

[15] A. J. Ferreri, M. Ponzoni, M. Guidoboni, et al., "BacteriaEradicating Therapy with Doxycycline in Ocular Adnexal MALT Lymphoma: A Multicenter Prospective Trial," Journal of the National Cancer Institute, Vol. 98, No. 19, 2006, pp. 1375-1382. doi:10.1093/jnci/djj373

[16] C. Garbe, H. Stein, D. Dienemann, et al., "Borrelia Burgdorferi-Associated Cutaneous B Cell Lymphoma: Clinical and Immunohistologic Characterization of Four Cases,” Journal of the American Academy of Dermatology, Vol. 24, No. 4, 1991, pp. 584-590. doi:10.1016/0190-9622(91)70088-J

[17] K. Lewis, C. Vandervelde, R. Grace, et al., "Salivary Gland Mucosa-Associated Lymphoid Tissue Lymphoma in 2 Patients with Sjogren's Syndrome: Clinical and Sonographic Features with Pathological Correlation,” Journal of Clinical Ultrasound, Vol. 35, No. 2, 2007, pp. 97101. doi:10.1002/jcu.20280

[18] R. Wozniak, L. Beckwith, H. Ratech, et al., "Maltoma of the Thyroid in a Man with Hashimoto's Thyroiditis," The Journal of Clinical Endocrinology \& Metabolism, Vol. 84, No. 4, 1999, pp. 1206-1209. doi:10.1210/jc.84.4.1206

[19] A. Sanjeevi, J. Krishnan, P. R. Bailey, et al., "Extranodal Marginal Zone B-Cell Lymphoma of Malt Type Involving the Cavernous Sinus,” Leukemia \& Lymphoma, Vol. 42, No. 5, 2001, pp. 1133-1137. doi:10.3109/10428190109097736

[20] T. Akagi, M. Motegi, A. Tamura, et al., “A Novel Gene, MALT1 at $18 \mathrm{q} 21$, Is Involved in $\mathrm{t}(11 ; 18)(\mathrm{q} 21 ; \mathrm{q} 21)$ Found in Low Grade B-Cell Ymphoma of Mucosa Associated
Lymphoid Tissue,” Oncogene, Vol. 18, No. 42, 1999, pp. 5785-5794. doi:10.1038/sj.onc.1203018

[21] B. Maes and C. de Wolf-Peeters, "Marginal Zone B Cell Lymphoma: An Update on Recent Advances," Histopathology, Vol. 40, No. S3, 2002, pp. 117-126. doi:10.1046/j.1365-2559.2002.01360.x

[22] L. Pasqualucci, O. Bereschenko, H. Niu, et al., "Molecular Pathogenesis of Non-Hodgkin's Lymphoma: The Role of Bcl-6,” Leukemia \& Lymphoma, Vol. 44, No. S3, 2003, pp. S5-S12. doi:10.1080/10428190310001621588

[23] J. Dierlamm, S. Pittaluga, M. Stul, et al., "BCL6 Gene Rearrangements Also Occur in Marginal Zone B-Cell Lymphoma,” British Journal of Haematology, Vol. 98, No. 3, 1997, pp. 719-725. doi:10.1046/j.1365-2141.1997.2703087.x

[24] N. Kambham, Y. Chang and A. Y. Matsushima, "Primary Lowgrade B-Cell Lymphoma of Mucosa-Associated Lymphoid Tissue (MALT) Arising in Dura,” Clinical Neuropathology, Vol. 17, 1998, pp. 311-317.

[25] M. Estevez, C. Chu, M. Pless, “ Small B-cell lym-phoma presenting as diffuse dural thickening with cranial neuropathies," Journal of Neuro-Oncology, Vol. 59, No. 3, 2002, pp. 243-237. doi:10.1023/A:1019913611512

[26] A. Garcia-Serra, M. N. Price, R. W. Hinerman, et al., "Management of Neurotropic Low-Grade B-Cell Lymphoma: Report of Two Cases," Head \& Neck, Vol. 25, No. 11, 2003, pp. 972-976. doi:10.1002/hed.10311

[27] N. L. Lehman, D. S. Horoupian, R. A. Warnke, et al., "Dural Marginal Zone Lymphoma with Massive Amyloid Deposition: Rare Low-Grade Primary Central Nervous System B-Cell Lymphoma. Case Report," Journal of Neurosurgery, Vol. 96, No. 2, 2002, pp. 368-372. doi:10.3171/jns.2002.96.2.0368

[28] W. Razaq, A. Goel, A. Amin and M. L. Grossbard, "Primary Central Nervous System Mucosa-Associated Lymphoid Tissue Lymphoma: Report and Literature Review," Clinical Lymphoma Myeloma and Leukemia, Vol. 9, No. 3, 2009, pp. E5-E9. doi:10.3816/CLM.2009.n.052 